\title{
Lung-inspired PEM fuel cells built from layered printed circuit boards
}

Authors: Viswanath Sasank B ${ }^{a, b}$, J.I.SCho ${ }^{a}$, Jennifer Hack ${ }^{b}$, D.J.L.Brett ${ }^{b}$ and M.-O. Coppens ${ }^{a}$

aEPSRC "Frontier Engineering" Centre for Nature Inspired Engineering \& Department of Chemical Engineering, University College London, London WC1E 7JE, United Kingdom

${ }^{b}$ Electrochemical Innovation Lab, Department of Chemical Engineering, University College London, London WC1E 7JE, United Kingdom

Abstract:

Homogenous, scalable, and uniform distribution of reactants at membrane-electrode assembly (MEA) level are critical to the performance of polymer electrolyte membrane (PEM) fuel cells. To achieve this, cathode flow fields were introduced that have fractal flow geometry, inspired by the air flow mechanism in lungs [1]. These were manufactured using direct metal laser sintering. Here, we discuss the design and characterisation of lunginspired fuel cell flow platesusing cheap and light printed circuit boards (PCBs) manufactured using computer numeric control (CNC) machining. X-Ray computed tomography (CT) scans of the developed fractal flow fields demonstrate a uniform and well aligned PCB plate assembly that results in a fractal hierarchy of flow channels, as shown in figure (1a) and (1b). Polarization performance at $70 \%$ reactants humidity and $45^{\circ} \mathrm{C}$ cell temperature for the fractal flow field was $12 \%$ higher than the performance of a singleserpentine, at $0.6 \mathrm{~V}$ individual cell potential as in figure (2). The cell temperature profile for the fractal flow field was more stable and uniform, compared to a rapid rise in profile of the single-serpentine one. The voltage decay measurements, at constant current density hold, indicatethat voltages of fractal and single-serpentine flow fields vary within $2 \%$ and $6 \%$ of their initial voltage values, respectively, over a period of $6 \mathrm{~h}$ continuous operation. Furthermore, Electrochemical Impedance Spectroscopic (EIS) measurements establish higher Ohmic and mass transport resistances for the single-serpentine compared to the fractal flow field, atcurrent densitiesranging between $100 \mathrm{~mA} / \mathrm{cm}^{2}$ and $900 \mathrm{~mA} / \mathrm{cm}^{2}$.

Keywords: Fractal, X-Ray CT, EIS,CNC, PCB, lung-inspired and hierarchical

Reference:

1 P. Trogadas, J.I.S. Cho, T.P. Neville, J. Marquis, B. Wu, D.J.L. Brett and M.-O. Coppens, 2018, A lung-inspired approach to scalable and robust fuel cell design. Energy \& Env. Sci. 11, 136-143.
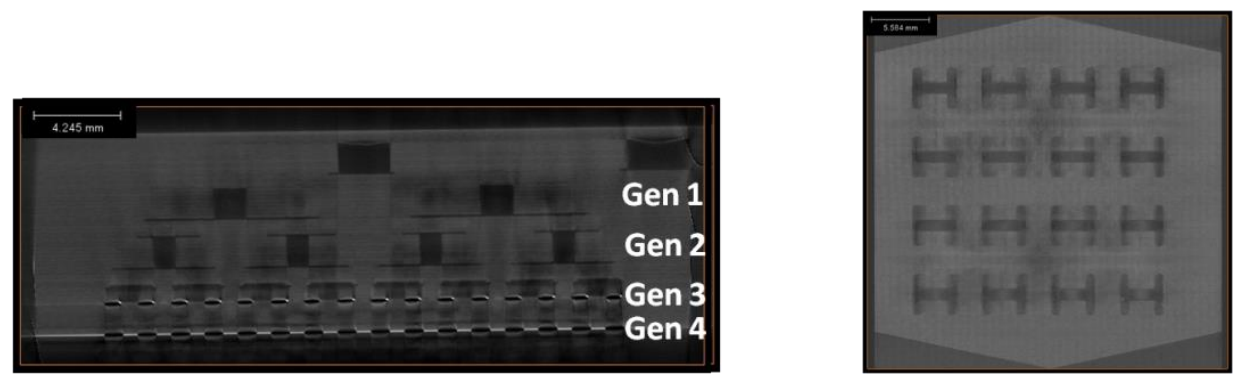